\title{
STIMULATED STRUCTURAL CHANGES OF SE IN NANOLAYERED COMPOSITE FILMS
}

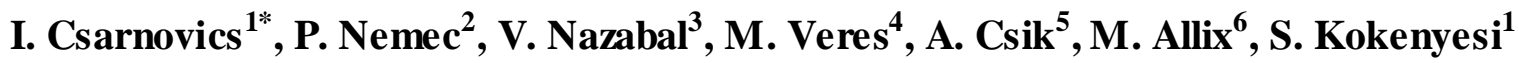 \\ ${ }^{1}$ Department of Experimental Physics, University of Debrecen, Bem sq. 18/a, 4026, Debrecen, Hungary \\ ${ }^{2}$ Department of Graphic Arts and Photophysics, Faculty of Chemical Technology, University of Pardubice, \\ Pardubice, Czech Republic \\ ${ }^{3}$ Equipe Verres et Céramiques, UMR-CNRS 6226, Sciences Chimiques de Rennes (SCR), Université de Rennes 1, \\ 35042 Rennes Cedex, France \\ ${ }^{4}$ Institute of Solid State Physics and Optics, Wigner RCP, HAS, Budapest, Hungary \\ ${ }^{5}$ ATOMKI, Debrecen, Hungary \\ ${ }^{6}$ CEMHTI-CNRS, Site Haute Température, Orléans, France
}

\begin{abstract}
Amorphous selenium (a-Se) is the simplest model material for investigation of the well-known photoinduced structural and optical transformations in amorphous chalcogenides. Since a lot of parameters and characteristics of materials essentially change at nanoscale, we applied nanostructuring to a-Se in nanomultilayers, obtained by pulsed laser deposition. The enhancement of photo- and thermally-induced crystallization was observed in a-Se/aluminosilicate nanomultilayers due to the initially heterogeneous state of Se in such samples, in comparis on with single a-Se layers.
\end{abstract}

Keywords : chalcogenides, multilayers, Raman spectroscopy and scattering, atomic force microscopy (AFM), crystallisation,

*Corresponding author, e-mail: csari86@gmail.com, tel. : +36-70-3314744, fax. : +36-52-315087 


\section{Introduction}

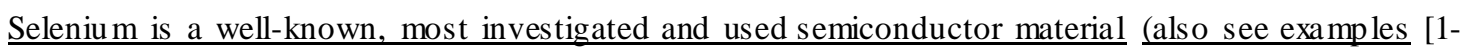
3]), which has a number of allotropic forms ( $\alpha$-, $\beta$-monoclinic, rhombohedral, trigonal, amorphous and glassy [4]). These forms can be comparatively easily transformed due to the unique flexibility of the structure, which may consist of eight-numbered rings, chains, separate molecules or their mixture. A morphous selenium (a-Se) films are among the simplest and the most easily fabricated (by low temperature vacuum evaporation)

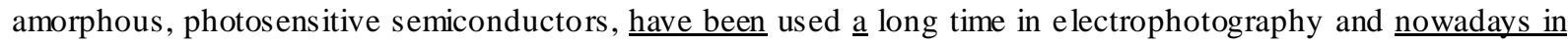
$\underline{X-r a y}$ imaging [5]. At the same time, a-Se films are rather unstable; they undergo structural transformations, crystallization during heating and/or illumination, which is obviously connected with the above mentioned structural peculiarities as well as with the presence of impurities and/or defects which enhance crystallization [6]. That is why a-Se may be considered as a simplest model material for investigation of the well-known and applied photoinduced structural/optical transformations in amorphous chalcogenides (see for example [7-10]). It exhibits photo-darkening, but the intense laser illumination or the thermal erasing of the darkening by annealing temperatures above the softening of a-Se $\left(\mathrm{T}_{\mathrm{g}} \approx 32^{\circ} \mathrm{C}\right)$ may result in crystallization [11].

Since a lot of parameters and characteristics of materials essentially change at nanoscale, it is interesting to apply nanostructuring to Se, especially taking into account the diversity of nano-dimensional frag ments composed of rings, chains and clusters. Quantum-size effect of blue-shift of the optical absorption edge, increased thermal stability as well as photo-induced interdiffusion were observed in a-Se/chalcogenide glass nanomultilayers (NMLs) like a-Se/As ${ }_{2} \mathrm{~S}_{3}$, obtained by cyclic thermal evaporation [12,13]. Thermally evaporated NMLs were amorphous as well as the amorphous solid solutions like As-S-Se glass resulting from the stimulated interdiffusion in such NMLs. The chalcogenide/chalcogenide NMLs, prepared by pulsed laser deposition (PLD) method usually exhibit the same photoinduced effects, interdiffusion under illumination. PLD makes possible to prepare NMLs from multicomponent chalcogenide glasses with higher softening temperatures (for example $\mathrm{GeS} / \mathrm{GeSe}$ ) but the whole structure seems to be more clustered due to the deposition conditions $[14,15]$.

In this paper we present investigations of pulsed laser deposited NMLs, which consist of few nanometers thick alumino-silicate (AS) diffusion-limiting barriers and a-Se light-sensitive layers, in which the structural transformations are es sentially stimulated. 


\section{Experimental}

The a-Se/AS NMLs were fabricated using bulk amorphous Se targets (typically of $99.999 \%$ purity) and AS hot-pressed targets. The AS polycrystalline targets were prepared from commercial kaolin in carbon matrix at $1100{ }^{\circ} \mathrm{C}$ under a pressure of $40 \mathrm{MPa}$, , It was the product of Ceske Lupkove zavody, Inc. It consists of alu mino-silicate with $61.9 \mathrm{w} \%$ of $\mathrm{O}, 16.8 \mathrm{w} \%$ of $\mathrm{A} 1$ and $21.3 \mathrm{w} \%$ of $\mathrm{Si}$.

For the fabrication of NMLs (75 a-Se/AS bi-layers), sequential PLD runs were employed using the above described targets. PLD setup consisted of a KrF excimer laser $(248 \mathrm{~nm}, 300 \pm 3 \mathrm{~mJ}$ per pulse, $30 \mathrm{~ns}$ pulse duration, $20 \mathrm{~Hz}$ repetition rate) and a vacuum chamber (background pressure $<2 \times 10^{-4} \mathrm{~Pa}$ ). The substrates used for PLD (chemically cleaned microscope glass slides, silicon wafers) were positioned parallel to the target surface which was $5 \mathrm{~cm}$ away from the substrates. The energy fluence on the target was changed with the

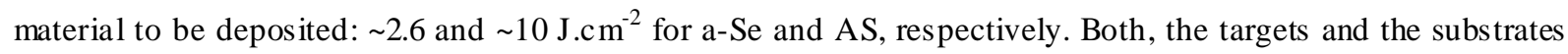
were rotated in off-axis geometry in order to avoid deep damage of the targets and to improve the thickness homogeneity of the films. The selected period $\Lambda=d(S e)+d(A S)$ was close to $9 \mathrm{~nm}$. Thus, the total thickness of the NML was expected be close to $700 \mathrm{~nm}$. For a comparison, single ( $400 \mathrm{~nm}$ thick) a-Se layers were deposited using PLD.

The quality and the periodicity of NMLs were controlled by low angle X-ray diffraction (LAXRD) [16]. The $\theta-2 \theta$ XRD measurements were carried out by a Siemens $\mathrm{Cu}$-anode $\mathrm{X}$-ray tube $\left(\lambda_{\mathrm{CuK} \alpha}=1.54 \AA\right)$ and a horizontal gonio meter equipped with graphite monochromator. LAXRD was used for the investigation of the heat and illumination effects on the periodicity of the sample. From the deposition rate data, the thicknesses of the individual sub-layers were obtained by dividing the total thickness of the separate control layers of the two components by the number of the bilayers.

Micro Raman-scattering method was used to follow structural transformations in single a-Se and NML samples. Raman scattering was measured by a Renishaw mic ro Raman spectrometer using a $785 \mathrm{n}$ m diode laser as excitation source.. The excitation beam was focused into a spot having diameter of around 2 microns. The intensity of the exciting light (being approx. $6 \mathrm{~mW}$ initially at the sample surface) was limited using neutral density filters in order to avoid damage and photostructural transformation in the samples. The integration time $\underline{\text { was } 100 \mathrm{~s} .}$

The total thickness of the NMLs were measured with Ambios XP-1 profilometer and Veeco diCaliber atomic force microscope (AFM). Used techniques confirmed the expected total thickness of the NML to be 
about $700 \mathrm{~nm}$. In case of single Se layers, the thickness was estimated to be about $400 \mathrm{~nm}$, which is comparable with the total thickness (sum) of the Se sub-layers in the NML structure. Surface roughness was also investigated by AFM, working in a tapping mode.

A transverse section of the sample was prepared perpendicular to the thin film surface to allow imaging the stacking of the different layers. The sampling was made by a Philips 200TEM Focused Ion Beam (FIB). The section has then been deposited onto a holey carbon film, supported by a copper grid. Imaging studies were then carried out with a Philips CM20 Trans mission Electron Microscope (TEM) operating at $200 \mathrm{kV}$.

The optical transmission spectra of the prepared samples were measured with Shimadzu UV-3600 spectrophotometer.

The investigated structures were irradiated by red $(\lambda=633 \mathrm{~nm}$, output power $\mathrm{P}=7 \mathrm{~mW})$ focused $\mathrm{He}-\mathrm{Ne}$ laser beam through a diaphragm with $1.2 \mathrm{~mm}$ diameter for 1 hour; the maximum light intensity at the surface was $600 \mathrm{~mW} / \mathrm{cm}^{2}$. The change of the transmission on the irradiation time has been detected with power meter setup (ThorLabs PM100).

\section{Results and discussion}

First, we were interested in the influence of laser irradiation on the surface, optical transmission spectra and structure of the samples. The NML and the single a-Se thin films, prepared by PLD were irradiated in a spot with a He-Ne laser. It was established, that the total thickness of the single a-Se layers increased for about $40 \mathrm{~nm}$ that is close to $10 \%$, which can be explained by the mechanism of the photo-induced lateral mass transport in chalcogenide glass, a-Se $[10,17]$, while there is no detectable volume change in NML. Although the AFM results have shown that the average roughness of the surface was increased due to laser illumination for NML (Fig.1).

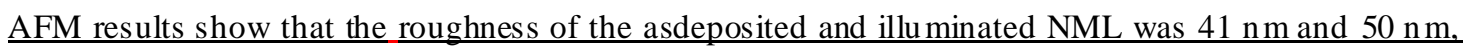
respectively, thus the increase was $9 \mathrm{~nm}$ or about $22 \%$ (with an error of $2 \mathrm{~nm}$ ). The roughness of the asdeposited single a-Se layer was $4 \mathrm{~nm}$, and it did not change due to the irradiation, but at the same time the thickness increased within the illu minated spot.

The transmission spectra of the single layers and NML samples were rather different and were influenced by laser irradiation (Fig. 2). The difference between the single a-Se layer and the NMLconsists in the lower transmission of the NML in the 550-1000 $\mathrm{nm}$ range; this is most probably caused by the increased 
scattering in NML due to the increased clustering and roughness. The last coincides with surface roughness values indicated by AFM.

The single layer undergoes slight photo-darkening during the irradiation, while one can observe photobleaching in case of the NML (Fig. 3). The change of the transmission $I$ relative to the initial one $I_{0}$ (at 633 $\mathrm{nm})$ with irradiation time is $\sim 0.95$ for the single layer in saturated state, while for the NML the increase reaches 1.25. The blue shift of the absorption edge in a-Se is known for a-Se/As ${ }_{2} S_{3}$ NML [12]. On the other hand, in our case the optical band gap $E_{g}$ determined from the intersection of $(\alpha h v)^{1 / 2}$ vs. $h v$ curve with $h v$ axis is smaller for NML as compared with a single a-Se (1.83 and $2.02 \mathrm{eV}$, correspondingly). This fact correlates with lower band gap energy of trigonal selenium $[3,6]$ and means that the NMLs fabricated by PLD should contain at least some part of crystalline Se phase, which was also supported by XRD and Raman scattering data, discussed below. That is why the blue shift quantum size effect is not observable, and the bleaching observed for the NMLs under study may be connected with further increase of the ordering under illu mination and decrease of the scattering in the given spectral region.

LAXRD and XRD measurements were performed on NMLs and single Se layers before and after irradiation (Figs. 4, 5). In the asdeposited NMLs, the intensity of the first diffraction peak is rather small and blurred, testifying the disordered structure of the NMLs (Fig. 4). The reason of the described behavior could be a) intermixing of the layers during the deposition, b) roughness on the interfaces inside the multilayer, which could be due to the presence of crystalline phases within material. Indeed, signs of some crystalline phase are visible in XRD spectra (Fig. 5) and in the Raman spectra of the NML and single Se layer samples indicating trigonal Se phase in the PLD samples.

After illumination, the LAXRD peaks (bands) intensity further decreases; the reason could be the change of ratio of the bilayers thickness ratio in the NMLs, which can be attributed to the increased of the crystalline phase content with higher density in the Se layers. This is shown by the Raman data as well.

The above mentioned XRD data taken before and after irradiation of the single Se layer and the NML are shown in Fig. 6. It is known that the trigonal selenium has peaks at the following angles: $23.57^{\circ}, 29.73^{\circ}$, $41.28^{\circ}, 43.68^{\circ}, 45.43^{\circ}, 51.72^{\circ}, 56.07^{\circ}, 61.62^{\circ}, 65.24^{\circ}$ and $\left.71.60^{\circ} \perp 18\right]$. For single Se layers, we have established the appearance of first two peaks, assigned to the 100 and 101 planes respectively. Intensity of these features increased after illu mination. The same behavior was observed in the spectra of the NMLs as well (Fig. 5).

Further, to compare the effect of irradiation and annealing, the samples were annealed at $110{ }^{\circ} \mathrm{C}$ for 30 minutes in vacuum (at this temperature, the influence of the increased stability, softening temperature of a-Se in 
thin sub-layers of a nanomultilayer vanishes [12]). A higher content of crystalline selenium phase in the asdeposited NMLs in comparis on with single selenium layers was established by XRD and Raman spectroscopy. During the heat treatment, crystallization was observed in both, NML and Se samples, but the resulting, annealing-induced crystallization was more significant in the single PLD seleniu m layer.

Comparison of the annealing- and irradiation-induced crystallization showed that the light accelerates the rate of the crystallization in the NMLs, while the heat treatment speeds up crystallization in the single selenium layer.

Micro-Raman scattering investigations provided further evidence of the crystallization processes taking place during deposition or treatments. It is well-known that Se has the following characteristic Raman features (bands): $248 \mathrm{~cm}^{-1}$ - amorphous selenium, $235 \mathrm{~cm}^{-1}$ - trigonal selenium, 80 and $112 \mathrm{~cm}^{-1}$ - ring-like selenium molecules, $138 \mathrm{~cm}^{-1}$ - helix-like seleniu m structural units [19]. In our experiments, the spectra were normalized to the largest intensity value and the changes in the relative intensities of the most characteristic bands were calculated. It was found that the intensity of the band related to amorphous Se is much higher in the asdeposited selenium films than in the NMLs. No band for trigonal crystalline phase $\left(235 \mathrm{~cm}^{-1}\right)$ was identified in the spectra of selenium layers, but in the case of NMLs such a feature was clearly observable (Fig. 6).

Five Raman peaks were identified in the spectra measured before and after illumination on NML samples (Fig. 6). The intensity of these bands change during laser treatment, especially those related to amorphous (at $\left.248 \mathrm{~cm}^{-1}\right)$ and trigonal $\left(235 \mathrm{~cm}^{-1}\right)$ seleniu $\mathrm{m}$ : the former decreases in comparison to the latter due to the illumination. At the same moment, Raman bands corresponding to ring-like and helix-like selenium structural units decrease slightly.

When analyzing the Raman spectra collected before and after the irradiation of the single selenium layer, we have again observed that due to illumination the intensity of characteristic peaks changes: the intensity of the peak of the amorphous selenium, ring-like and helix-like selenium decreased and the peak (shoulder) at $235 \mathrm{~cm}^{-1}$ became more pronounced.

In the case of single selenium layer, the changes in the spectrum after light treatment were not so remarkable: slight increase of the band attributed to trigonal Se (at $235 \mathrm{~cm}^{-1}$ ) was observed, together with the intensity decrease of peaks connected with ring-like and helix-like selenium. The last may be related to the increased scattering at very low wavenumbers, in the region of the the so called Boson peak, which also means increased clustering and mediu m range ordering in glasses. 
All the above presented data indicate that the basic structural transformation process es induced in a-Se layers by laser irradiation or by annealing are related to the formation of trigonal crystalline phase; such effects are enhanced in the PLD a-Se/AS NMLs. This idea is also supported by increasing surface roughness (and related clustered structure of the PLD samples), observed in the AFM data depicted in Fig. 1. The crystalline phase is directly visible on the TEM pictures of cross-sections (Fig. 7). That means formation of larger, about 10 nm crystalline clusters during the highly non-equilibrium PLD process of NML fabrication, which is absent in aSe films prepared by more equilibriu m process of thermal evaporation and even in the single layer fabricated by PLD.

Besides the well-known photo-darkening, the light induces also thickness change occurring in a single a-Se layer, which can be followed by a mass transport in the presence of non-homogeneous illu mination [9], but, as one can see, in certain samples and at certain conditions, the enhancement of the crystallization processes can occur which overlaps mass transport phenomenon. Thus, the precise measurements of the induced thickness change $(\Delta \mathrm{d})$ in the layers with enhanced crystallization are impeded. We got for the single selenium layer $\Delta \mathrm{d}$ of about $+40 \mathrm{~nm}$ i.e. $\approx 10 \%$ increase (we observed hillocks and valleys - it can be concluded that the photo-induced giant mass transport is present), while for the NML we could not measure the exact value for the thickness change because of the too high value of the surface roughness before and after the treatment. As already mentioned, this result can be explained by the crystallization process that occurs in the NMLs. Due to the crystallization, it is difficult to evaluate precisely the change of the refractive index and/or the absorption coefficient. In addition, the variation of thickness is strongly suppressed by the presence of hard barriers in NMLs, as it was shown for $\mathrm{As}_{20} \mathrm{Se}_{80} / \mathrm{AS}$ NMLs [20].

\section{Conclusions:}

From the results presented in this work, it is evident that the PLD method of fabrication of thin amorphous chalcogenide nanolayered structures which consist of components with large difference in ablation pulse energy (low for a-Se, high for AS), gives highly metastable films or structures being farther from equilibrium than structures prepared using thermal vacuum evaporation. Structural transformations can be enhanced in these materials, especially the heterogeneous nucleation and crystallization may occur due to the presence of initial crystalline seeds and additional interfaces in NMLs. It was shown earlier by Tallman [21] and Abbaszadeh [22], that the interface has an effect on the photo-induced metastability in a-Se structures. It was established by them that the crystallization in a-Se is driven by the local strains, which are enhanced at the 
interfaces (including crystalline-amorphous interface, selenium-substrate interface or interface in multilayered structures). In addition, the crystallization rate increases as a result of enhancing influence of the decreasing viscosity under illu mination or heating (so called photo-plastic effect in amo rphous chalcogenides [17]).

Application of the PLD technology to chalcogenide-based NMLs gives clustered film structures with higher roughness (as it was established in $\mathrm{GeSe} / \mathrm{GeS}$ NMLs too) [13]. If one component (in our case $\mathrm{Se}$ ) undergoes crystallization easily, the process takes place and the role of initial clustered structures in the crystallite formation is enhanced by the heterogeneous nucleation in the Se sub-layers and at the interfaces with oxide barriers, which are created under higher laser pulse energies as the Se sub-layers. The increase of the thickness seems to contradict the decrease of the Se volume during crystallization, but in the PLD samples the general, macroscopic disorder increas es making the whole structure more heterogeneous.

\section{Acknowledgements}

This work was supported by the TAMOP 4.2.1./B-09/1/KONV-2010-0007, TAMOP 4.2.2./B-10/12010-0024 and TAMOP 4.2.2.A-11/2/KONV-2012-0032 projects, which are co-financed by the European Union and European Social Fund. The financial support of the Czech Science Foundation under the project No. 13$05082 \mathrm{~S})$ is acknowledged.

\section{References:}

[1] P. Unger, P. Cherin, Physics of Se leniu m and telluriu m, ed. W. C. Cooper, Pergamon, Oxford, 1969.

[2] W. C. Cooper, R. A. Westbury, Seleniu m, ed. R. A. Zingaro and W. C. Cooper, Reinhold, New York, 1974.

[3] V. V. Sobolev, A. M. Sh irokov, Electron structure of chalcogens, Nauka, Moscow, 1988.

[4] V. S. Minaev, S. P. Timoshenkov, V.V. Kalugin, J. Optoelectron. Adv. Mater. 4 (2005) 1717-1741.

[5] S. Kasap, P. Copper, Springer Handbook of Electronic and Photonic Materials, Springer, Berlin, 2006.

[6] A. Feltz, A morphe und Glasartige Anorganishe Festkörper, Academie Verlag, Berlin, 1983.

[7] Mihai A. Popescu, Non-Crystalline chalcogenides, Kluver Academic Publisher, Durdrecht, 2000.

[8] A. V. Kolobov, Photo-induced Metastability in Amorphous Semiconductors, Wiley-VCH, Weinheim $\underline{2003 .}$

[9]A. Kikineshi, V. Palyok, M. Shiplyak, I. A. Szabo, D. Beke, J. Optoelectron. Adv. Mater. 2 (2000) 9598.

[10] M. L. Trunov, P. M. Lytvyn, S. N. Yannopoulos, I. A. Szabo, S. Kokenyesi Appl. Phys. Lett. 99 (2011) 051906.

[11] A. Kikineshi, V. Fedak, V. Stephanovich, J. Nauch. Prikl. Fotografii i Cinematogr. 31 (1986) 433-435.

[12] A. Kikineshi., A. Mishak, V. Palyok, M. Shipljak, Nanostructured Materials, 12 (1999) 417-420.

[13] K. V. Adarsh, R. Naik, K. S. Sangunni, S. Kokenyesi, H. Jain, A. C. Miller, J. Appl. Phys. 104 (2008) 053501.

[14] P. Nemec, V. Takats, A. Csik, S. Kokenyesi, J. Non-Cryst. Solids 354 (2008) 5421-5424.

[15] V. Takats, P. Nemec, A. Csik, S. Kokenyesi, J. Phys. Chem. Solids 68 (2007) 948-952.

[16] A.L. Greer, F. Spaepen, in: L.L. Chang, B.C. Giessen (Eds.), "Diffusion", Synthetic Modulated Structures, Academic Press, New York, 1985, pp. 419-486.

[17] S. N. Yannopoulos, M. L. Trunov, Phys. Status Solidi B 246 (2009) 1773.

[18] C. K. Senthil Kumaran, S. Agilan, Dhayalan Velauthapillai, N.Muthuku marasamy, M. Thambidurai, T. $\underline{\text { S. Senthil, R. Balasundaraprabhu, International Scholarly Research Network, ISRN Nanotechnology (2011) }}$ doi:10.5402/2011/589073.

[19] S. N. Yannopoulos, K. S. Andrikopoulos, J. Chem. Phys. 121 (2004) $4747-4759$.

[20] S. Charnovych, P. Nemec, V. Nazabal, A. Csik, M. Allix, G. Matzen, S. Kokenyesi, Mat. Chem. Phys. $130(2011) 1022-1025$. 
[21] R.E. Tallman, B. A. Weinstein, A. Reznik, M. Kubota, K. Tanioka, J. A. Rowlands, J. Non-Cryst. Solids 354 (2008) 4577-4581.

[22] S. Abbaszadeh, K. Rom, O. Bubon, B. A. Weinstein, K. S. Karim, J. A. Rowlands, A. Reznik, J. NonCryst. Solids 358 (2012) 2389-2392.

\section{Figures}
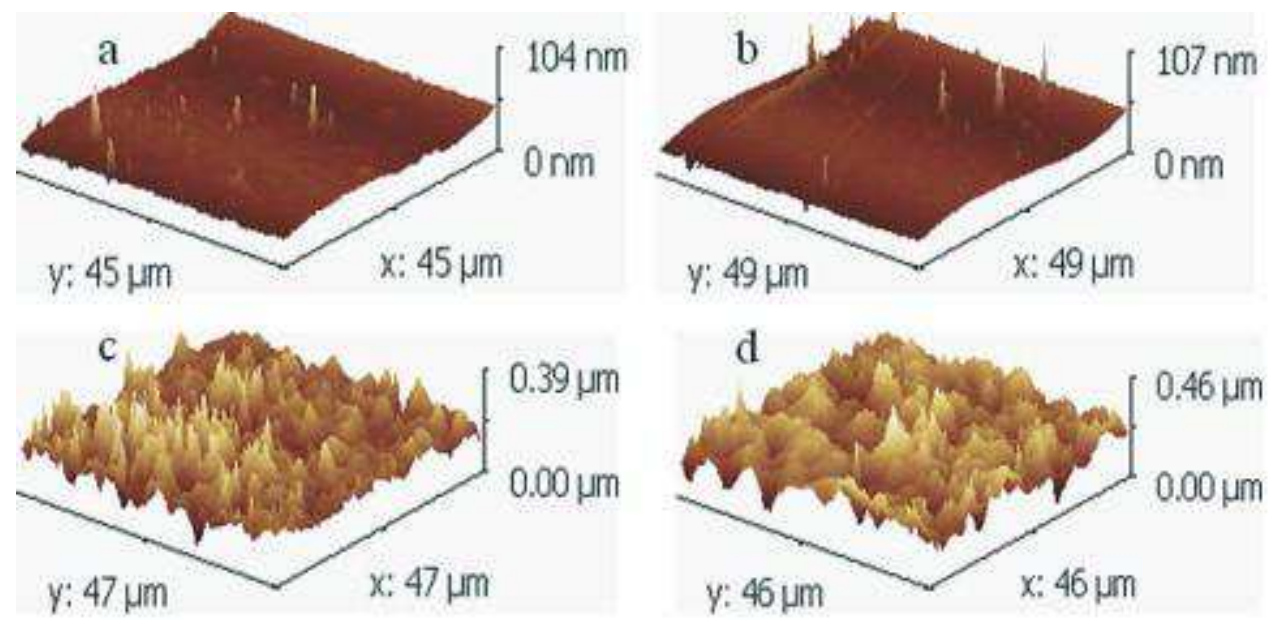

1. AFM pictures of the single selenium layer ( $a, b)$ and NML (c, d) before (left images) and after (right images) illumination.

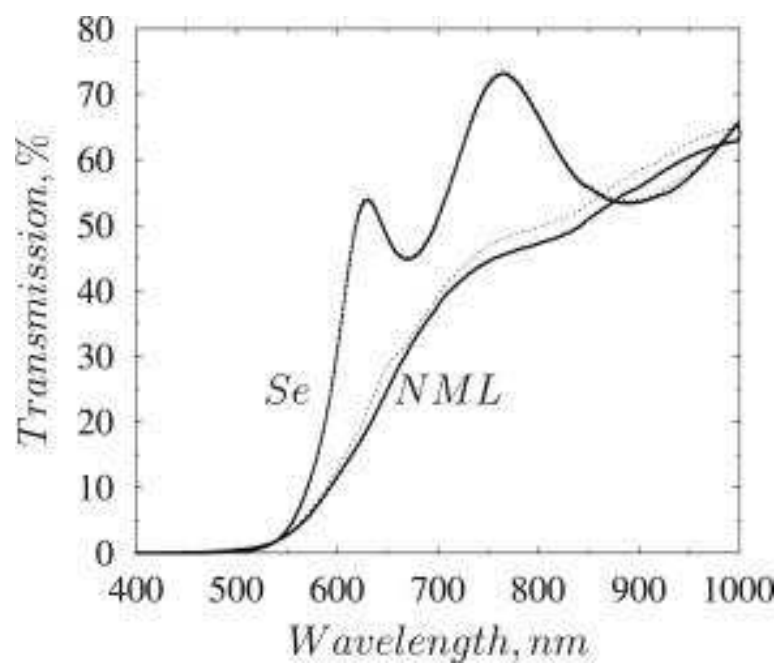

2. Optical transmission spectra of single a-Se layer and NML samples before (single line) and after illumination (dash line). 


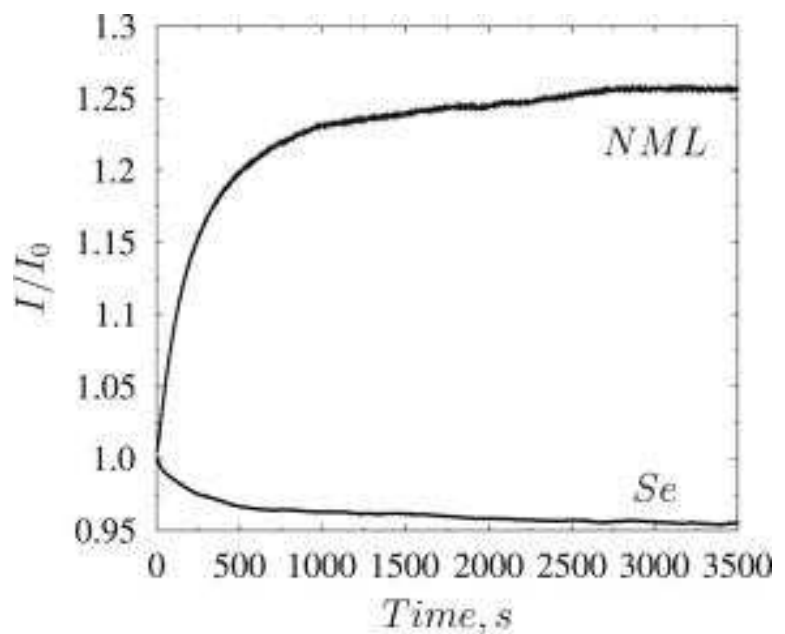

3. Change of the relative optical trans mis sion at $633 \mathrm{~nm}$ under continuous laser illumination in single a-Se thin film (1) and a-Se/AS NML (2).

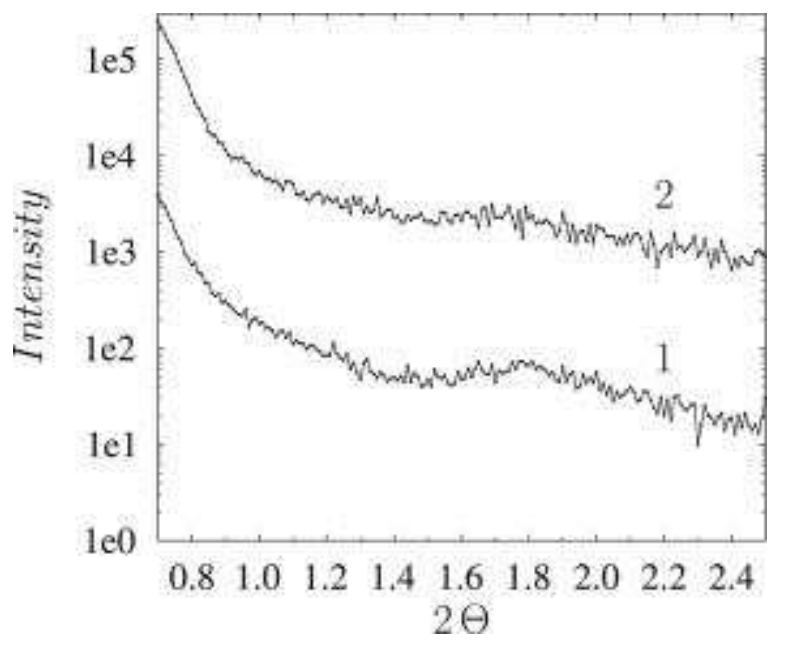

4. LAXRD of Se/As NML before (1) and after (2) laser irradiation.
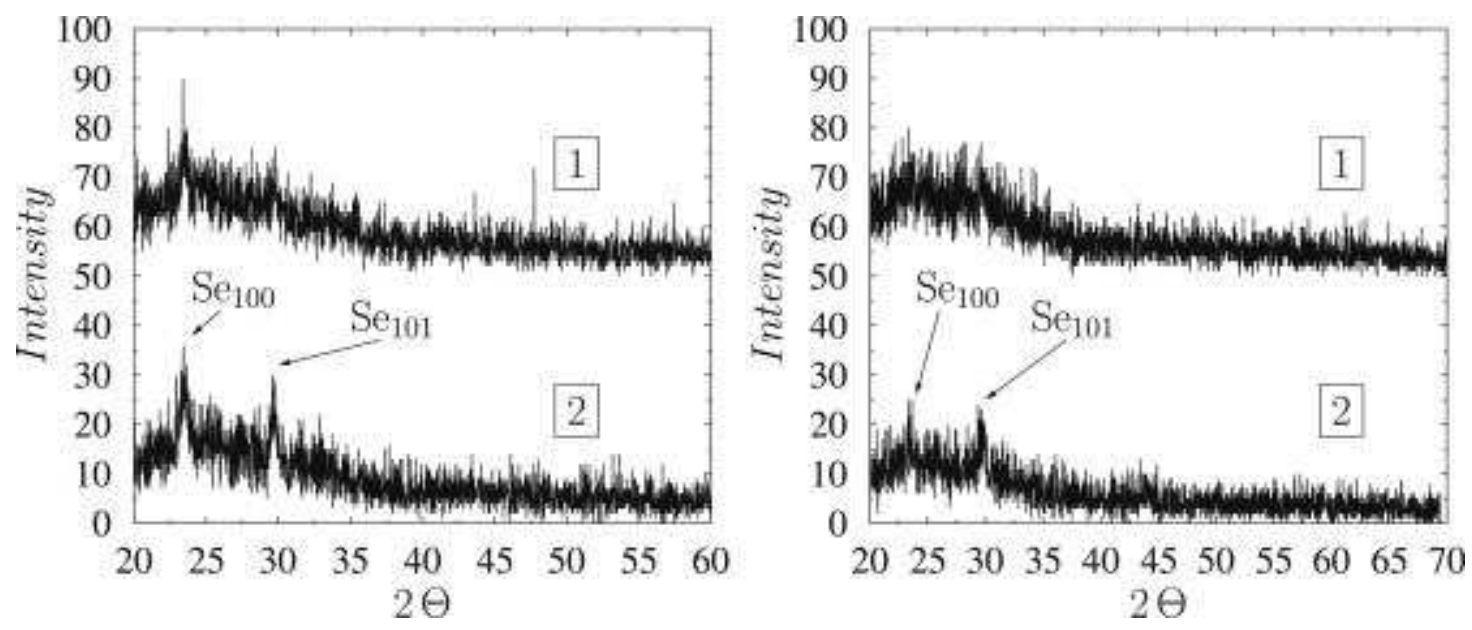

5. X-ray diffractograms of Se/As NML (left) and single a-Se layer (right) before (1) and after (2) illumination. 

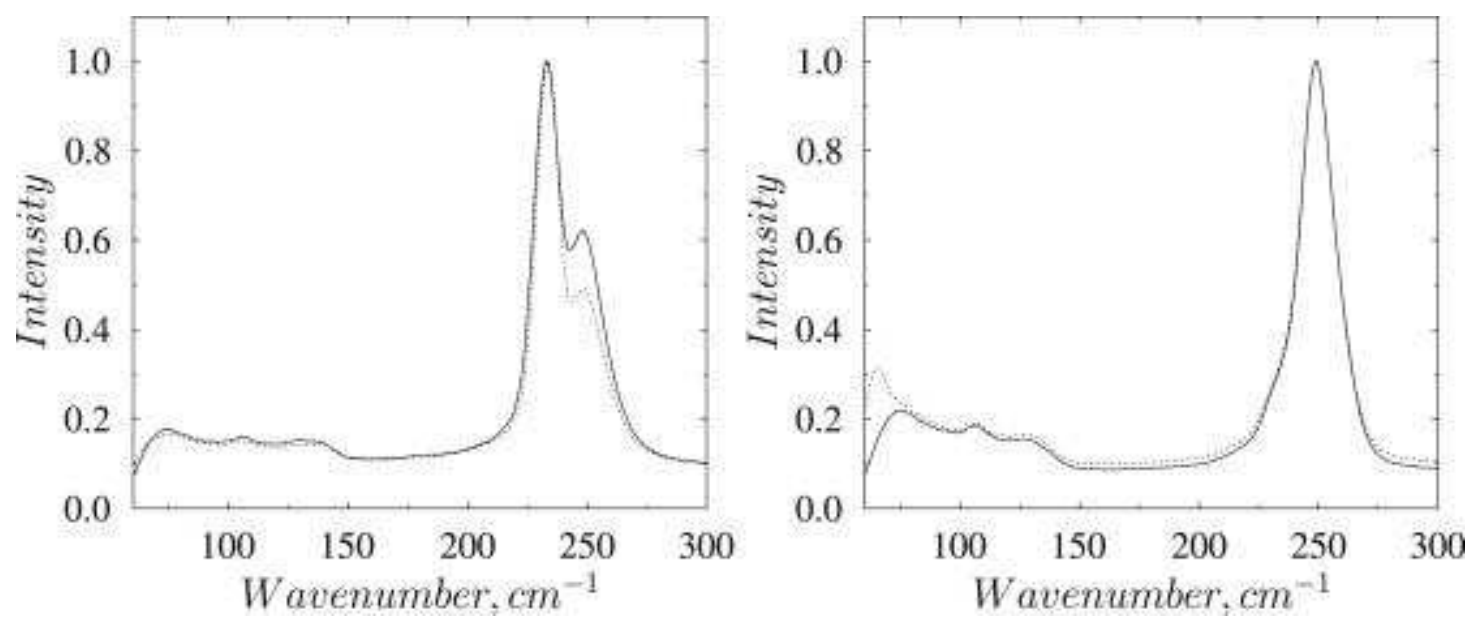

6. Raman spectra of Se/AS NML (left) and single a-Se layer (right) sample before (single line) and after (dot line) illumination.

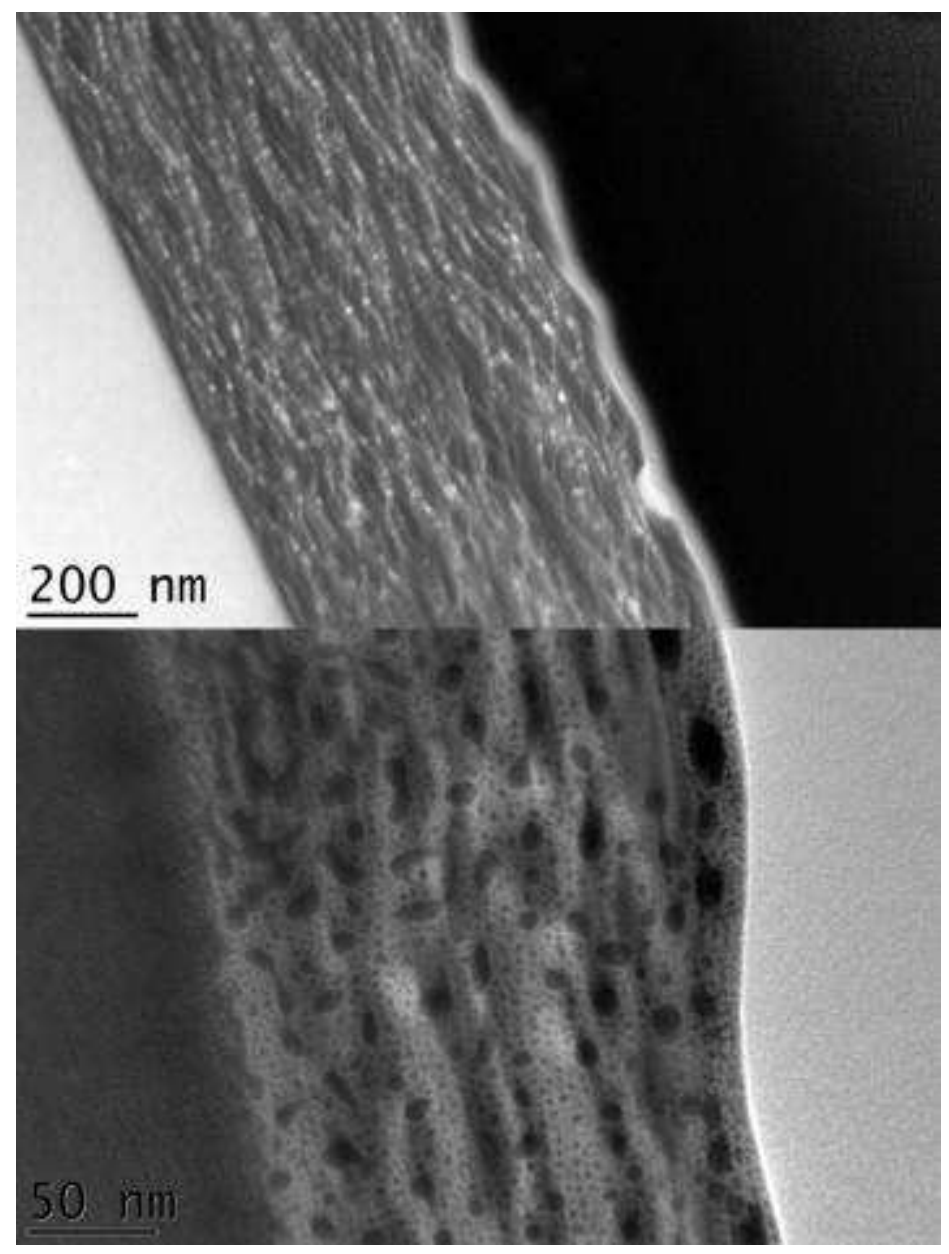

7. TEM photographs (with different magnification) of the cross-section of PLD a-Se/AS NML in as-deposited state. Lighter color tone corresponds with AS, darker color tone is then related with selenium. Note that the structure periodicity is partly lost due to electrons with high energy used by TEM instrument. 